\title{
Proteomic analysis of extracellular vesicles produced by placental mesenchymal stem cells
}

\author{
Anastasia Khutornenko \\ Laboratory of Cell Technologies, \\ Research Center for Obstetrics, \\ Gynecology and Perinatology, \\ Moscow, Russia \\ bioingenier@gmail.com
}

Sergey Kovalchuk Laboratory of Bioinformatic methods for Combinatorial Chemistry and Biology, Shemyakin-Ovchinnikov Institute of Bioorganic Chemistry, Moscow, Russia xerx222@gmail.com

Egor Plotnikov

A.N. Belozersky Institute of PhysicoChemical Biology; Research Center of Obstetrics, Gynecology and Perinatology,

Moscow, Russia

plotnikov@belozersky.msu.ru

\author{
Anastasia Zharikova \\ Faculty of Bioengineering and \\ Bioinformatics, \\ Lomonosov Moscow State University, \\ Moscow, Russia \\ azharikova89@gmail.com \\ Kirill Goryunov \\ Laboratory of Cell Technologies, \\ Research Center for Obstetrics, \\ Gynecology and Perinatology, \\ Moscow, Russia \\ kirishgor@gmail.com
}

Dmitry Zorov

A.N. Belozersky Institute of PhysicoChemical Biology; Reserch Center of Obstetrics, Gynecology and Perinatology,

Moscow, Russia

zorov@belozersky.msu.ru

\author{
Vasily Popkov \\ The A.N. Belozersky Institute Of \\ Physico-Chemical Biology, \\ Lomonosov Moscow State University, \\ Moscow, Russia \\ popkov.vas@gmail.com \\ Yulia Shevtsova \\ Laboratory of Cell Technologies, \\ Research Center for Obstetrics, \\ Gynecology and Perinatology, \\ Moscow, Russia \\ yulshevtsova@yandex.ru
}

Denis Silachev

A.N. Belozersky Institute of PhysicoChemical Biology; Research Center of Obstetrics, Gynecology and Perinatology

Moscow, Russia

d_silachev@oparina4.ru

\begin{abstract}
Mesenchymal stem/stromal cells (MSCs) have therapeutic potential in many pathological conditions, that is explained mostly by their paracrine action. MSCs secrete extracellular vesicles (EVs) packed with proteins, DNA, RNA and lipids. One of the easily accessible and reach sources of MSCs is placenta. In order to reveal possible pathways, by which EVs produced by placenta-derived MSCs (EVs-PMSC) could realize their therapeutic potential we conducted proteomic analysis of EVs-PMSC and subsequent bioinformatic analysis of identified proteins using Reactome pathway database. Identified peptides were mapped to 2093 proteins. Among the 2093 proteins, 972 proteins were identified in at least four LC-MS/MS analyses out of eight. According to Reactome the most significant 'top three' 1st level pathways for detected EVsPMSC proteins were 'Cellular responses to external stimuli'; 'Immune system'; 'Developmental biology'. Some of the most enriched pathways in the group 'Cellular responses to external stimuli' were: 'Response of EIF2AK4 (GCN2) to amino acid deficiency'; 'Cellular response to hypoxia'; 'Detoxification of Reactive Oxygen Species'. Some of the most enriched pathways in the group 'Immune System' were: 'Class I MHC mediated antigen processing \& presentation'; 'Neutrophil degranulation'; 'Interferon Signaling'. The most enriched pathway in the group 'Developmental biology' was 'Axon guidance'. Thus far, concerning most enriched pathways EVs-PMSC proteome is expected to: 1) provide protection against stress (amino acid deficiency, hypoxia, ROS) to the damaged tissues; 2) possess immunomodulation properties; 3 ) facilitate axon guidance. The result (the top list of enriched pathways) leads to the notion that EVs-PMSC could be effective in terms of neuroprotection.
\end{abstract}

Keywords - extracellular vesicles, mesenchymal stem cells, placenta, proteomic analysis, reactome analysis

\section{Introduction}

It is widely accepted that the therapeutic potential of mesenchymal stem/stromal cells (MSCs) is attributed mostly to their paracrine activity [1], [2]. MSCs secrete extracellular vesicles (EVs) (exosomes, microvesicles, apoptotic bodies) packed with signaling molecules, proteins, DNA, RNA, lipids, all necessary for short and long distance intercellular communication [1]. One of the easily available and reach sources of MSCs is placenta tissue. In order to reveal possible pathways, by which EVs produced by placenta-derived MSCs (EVs-PMSC) could realize their therapeutic potential we conducted proteomic analysis of EVs-PMSC and subsequent bioinformatic analysis of identified proteins using Reactome pathway database. Identified peptides were mapped to 2093 proteins. Among the 2093 proteins, 972 proteins were identified in at least four LC-MS/MS analyses out of eight. Most enriched pathways revealed by Reactome analysis for identified proteins were related to cell stress response (response to hypoxia, reactive oxygen species, heat), immune system, axon guidance. The result leads to the notion that EVsPMSC could effective in terms of neuroprotection.

\section{Materials and Methods}

\section{MSC culture}

Fresh human preterm placenta samples $(n=4)$ were obtained from healthy women 25 to 30 years old at the V.I. Kulakov National Medical Research Center for Obstetrics, Gynecology, and Perinatology, according to the World Medical Association Declaration of Helsinki and with the permission of the local ethics committee, informed consent was obtained from all subjects.

MSCs were isolated from tissue samples and cultivated as previously described [2]. MSCs were cultivated as adhesive cultures in DMEM/F12 medium (Paneco, Moscow, Russia) containing 7\% FBS (Biosera, Nuaille, France). 
Isolation of EVS

EVs were isolated from MSCs conditioned medium by differential centrifugation, and analyzed by nanoparticle tracking analysis (NTA), and transmission electron microscopy (TEM) as described previously [2].

\section{Proteomic analysis}

Sample preparation was performed as in [3] with modifications. LC-MS analysis was performed on an Ultimate 3000 RSLCnano HPLC system connected to a QExactive Plus mass spectrometer (ThermoFisher Scientific) in DDA mode. Each sample was analyzed in two technical repeats. Protein identifications and data analysis were done in MaxQuant and Perseus.

A set of identified 972 proteins was used for Reactome analysis (www.reactome.org).

\section{Results}

MSCs were isolated from human preterm placenta samples. The cell viability was assessed by trypan blue exclusion (generally $>95 \%$ ). MSCs used in our study were positive for mesenchymal stem cell markers (CD73, CD90, CD105) and negative for hematopoietic cell markers (CD14, CD20, CD45, CD34). MSCs at the third passage were used for EVs isolation. According to NTA, EVs showed a broad size distribution $(40-250 \mathrm{~nm})$, which indicates the presence of both exosomes and microvesicles. TEM of EV preparations confirmed two types of particles (exosomes and microvesicles). The majority of objects showed a cup-shape morphology characteristic of EVs, with sizes ranging from 40 to $300 \mathrm{~nm}$. A minor fraction of the smaller objects $(27-95 \mathrm{~nm})$ had a smooth or angulated shape.

Isolated EVs-PMSC were used for proteomic analysis. 972 proteins were identified and analyzed using Reactome pathway database. Top three enriched pathways are provided in Table I (dark grey).

TABLE I. Reactome analysis of EVs-PMSC proteome

\begin{tabular}{|l|c|c|}
\hline \multicolumn{1}{|c|}{ Pathway identifier } & FDR & pValue \\
\hline Cellular responses to external stimuli & $3.89 \mathrm{E}-15$ & $1.11 \mathrm{E}-16$ \\
\hline $\begin{array}{l}\text { Response of EIF2AK4 (GCN2) to amino acid } \\
\text { deficiency }\end{array}$ & $3.89 \mathrm{E}-15$ & $1.11 \mathrm{E}-16$ \\
\hline Cellular response to hypoxia & $2.41 \mathrm{E}-06$ & $3.01 \mathrm{E}-07$ \\
\hline $\begin{array}{l}\text { Detoxification of Reactive Oxygen Species } \\
\text { (ROS) }\end{array}$ & $1.49 \mathrm{E}-03$ & $3.72 \mathrm{E}-04$ \\
\hline $\begin{array}{l}\text { Immune system } \\
\text { Class I MHC mediated antigen processing \& } \\
\text { presentation }\end{array}$ & $3.89 \mathrm{E}-15$ & $1.11 \mathrm{E}-16$ \\
\hline Neutrophil degranulation & $3.89 \mathrm{E}-15$ & $1.11 \mathrm{E}-16$ \\
\hline Interferon Signaling & $3.89 \mathrm{E}-15$ & $1.11 \mathrm{E}-16$ \\
\hline Developmental biology & $3.89 \mathrm{E}-15$ & $1.11 \mathrm{E}-16$ \\
\hline Axon guidance & $3.89 \mathrm{E}-15$ & $1.11 \mathrm{E}-16$ \\
\hline
\end{tabular}

The group 'Response of EIF2AK4 (GCN2) to amino acid deficiency' includes ribosomal proteins and GCN1 (eIF-2alpha kinase activator GCN1), a positive activator of the EIF2AK4/GCN2 protein kinase activity in response to amino acid starvation (www.uniprot.org).
The group 'Cellular response to hypoxia' includes some components/proteins required for HIF1a proteasomal degradation, i.e. proteasomal subunits, elongin $\mathrm{B}$ and $\mathrm{C}$, polyubiquitin $\mathrm{B}$ and $\mathrm{C}$.

The group 'Detoxification of ROS' includes Peroxiredoxins (PRDX1, 2, 3, 5, 6), Thioredoxin (TXN), Thioredoxin reductases 1 and 2 (TXNRD1, 2), Glutathione Stransferase P (GSTP1), protein disulfide isomerase (PDIA1), superoxide dismutase (SOD1).

The group 'Axon guidance' includes 178 of identified proteins, and can be subdivided into following enriched pathways: 'Signaling by ROBO receptors' ( $p$-value 1,11E16); 'L1CAM interactions' (p-value 7,77E-16); 'EPH-Ephrin signaling' (p-value 3,48E-13); 'Semaphorin interactions' ( $p$ value 4,01E-06). Identified proteins are implicated in cytoskeleton dynamics/organization (necessary for growth cone formation), synaptic plasticity, adhesion to extracellular matrix.

The group 'MHC-I mediated antigen processing and presentation' among 114 proteins contains HLA-F, nonclassical major histocompatibility class Ib molecule postulated to play a role in immune surveillance, immune tolerance and inflammation; may play a role in inflammatory responses in the peripheral nervous system; through interaction with KIR3DL2, may protect motor neurons from astrocyte-induced toxicity (www.uniprot.org); HLA-C; HLA-A; HLA-B; HLA$\mathrm{H}$; B2M.

The group 'Neutrophil degranulation' includes 117 of identified proteins involved in the regulation of neutrophil functioning. As an example of protein influencing neutrophil transendothelial migration is Annexin A2 (ANXA2).

The group 'Interferon signaling' includes 89 of identified proteins, all of them downstream of IFN, with STAT1 as a main player of IFN-induced genes expression.

\section{DISCUSSION}

In terms of neuroprotection activation of EIF2AK4/GCN2 [4], degradation of HIF1a [5], detoxification of ROS, axon guidance are favorable processes. Immune reactions, like MHC-I expression [6], neutrophil degranulation [7], interferon signaling [8], may have dual effect: protective by modulating synaptic plasticity, axonal regeneration, tissue repair and regeneration; destructive by playing neuroinflammatory role.

\section{ACKNOWLEDGMENT}

This study was supported by the Russian Foundation for basic research 20-015-00414.

\section{REFERENCES}

[1] K.-S. Park et al. Stem Cell Res Ther, vol. 10, no. 1, p. 288, Sep. 2019, doi: 10.1186/s13287-019-1398-3.

[2] D. N. Silachev et al. Cells, vol. 8, no. 3, 192019.

[3] S.I. Kovalchuk et al. Mol Cell Proteomics, vol. 18, no. 2, pp. 383-390, Feb. 2019, doi: 10.1074/mcp.TIR118.000953.

[4] D.W. Gietzen and S.M. Aja. Mol. Neurobiol., vol. 46, no. 2, pp. 332348, Oct. 2012, doi: 10.1007/s12035-012-8283-8.

[5] H. Shi. Curr. Med. Chem., vol. 16, no. 34, pp. 4593-4600, 2009, doi: 10.2174/092986709789760779.

[6] C. Cebrián, J. D. Loike, and D. Sulzer. Front Neuroanat, vol. 8, p. 114 2014, doi: 10.3389/fnana.2014.00114.

[7] J. Wang. Cell Tissue Res, vol. 371, no. 3, pp. 531-539, Mar. 2018, doi: 10.1007/s00441-017-2785-7.

[8] A. McDonough, R. V. Lee, and J. R. Weinstein. Neurochem. Res., vol. 42, no. 9, pp. 2625-2638, Sep. 2017, doi: 10.1007/s11064-017-2307-8. 